\title{
Trazando puentes entre las neurociencias y la educación. Aportes, límites y caminos futuros en el campo educativo
}

\author{
Building bridges between neuroscience and education. \\ Neurosciences' contributions, limitations and future \\ directions in the education field
}
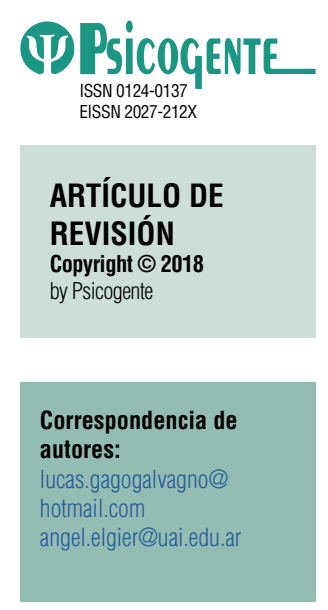

Recibido: 06-03-17 Aceptado: 29-09-17 Publicado: 01-07-18

\author{
Lucas G. Gago Galvagno (iD \\ Universidad Abierta Interamericana. Buenos Aires, Argentina \\ Ángel M. Elgier (iD) \\ Universidad de Buenos Aires, CONICET. Buenos Aires, Argentina
}

\section{Resumen}

Las neurociencias son el conjunto de disciplinas cuyo objetivo de investigación es el sistema nervioso, poniendo el acento en la actividad del cerebro y su relación con nuestros comportamientos. Abordan aspectos neurobiológicos de la conducta y se apoyan en la psicología cognitiva, la lingüística, la antropología y la inteligencia artificial. La neuroeducación, también llamada neurociencia educativa, es una disciplina en expansión que se ocupa de estudiar la optimización del proceso de enseñanza y aprendizaje con base en el funcionamiento del cerebro y los fundamentos neurobiológicos que lo sustentan. El artículo se propone revisar aportes de las neurociencias al campo educativo, y a su vez críticas y futuros caminos para establecer relaciones aún más estrechas entre ambos. Se concluye que a pesar de que los aportes de la neuroeducación son variados y se esperan avances en el área, derivados de nuevas tecnologías, debemos ser cautos y críticos a la hora de analizarlos o ponerlos en práctica, ya que aún se deben generar estudios con mayor validez ecológica, en entornos educativos con seres humanos y con distintos niveles de análisis, sin dejar de lado la interdisciplina.

\section{Palabras clave: neurociencias, neuroeducación, educación, aprendizaje.}

\section{Abstract}

Neurosciences are a set of disciplines which aims to research the nervous system, focusing on brain functioning in relation with our behaviors. Neurosciences not only address the neurobiological issues of behavior but also rely on cognitive psychology, linguistics, anthropology and artificial intelligence. Neuroeducation, also called educational neuroscience, is an expanding discipline that aims to study brain development and its neurobiological foundations by optimizing teaching and learning process. Through analyzing some classic and current contributions of the neurosciences to the educational area, this paper proposes to review neurosciences' contribution to the educational field, establishing at the same time critical and future ways to get even closer relations between them. Then, we conclude that although the neuroeducation contributions are varied and we expect advances in this area derived from new technologies, we must be cautious and critical when analyzing or putting them into practice, since research must be generated with greater ecological validity, in educational environments with human beings with analysis at different levels, without ignoring the interdiscipline.

Key words: neuroscience, neuroeducation, education, learning

Cómo citar este artículo (APA):

Gago Galvagno, L. G. \& Elgier, A. M. (2018). Trazando puentes entre las neurociencias y la educación. Aportes, límites y caminos futuros en el campo

educativo. Psicogente 21(40), 476-494. https://doi.org/10.17081/psico.21.40.3087 


\section{INTRODUCCIÓN}

Las neurociencias nacen como un paraguas epistemológico que reúne a diferentes disciplinas (física, psicología, filosofía, medicina, biología, química, entre otras) con la intención de conocer la estructura, la función, el desarrollo, la bioquímica, el funcionamiento neuronal y la patología del sistema nervioso, así como la forma en que sus diferentes elementos interactúan, dando lugar a las bases biológicas de la conducta (Blakemore \& Frith, 2011). De esta forma, se puede establecer el correlato neurobiológico de los comportamientos y funciones cognitivas humanas, desarrollando nuevas formas de comprender la mente. La aplicación de esta disciplina al campo educativo constituye la llamada neurociencia educativa o neuroeducación.

La neuroeducación se ocupa de estudiar la optimización del proceso de enseñanza y aprendizaje con base en el desarrollo del cerebro y los fundamentos neurobiológicos que lo sustentan. La misma está conformada por numerosas disciplinas y promueve una mayor integración de las ciencias de la educación con aquellas que se ocupan del desarrollo neurocognitivo y el funcionamiento cerebral del ser humano (Battro \& Cardinali, 1996). Es una disciplina que se encuentra en plena construcción como resultado del entrecruzamiento de los aportes de las neurociencias y de las ciencias de la educación (Paterno, 2014).

Mediante distintas técnicas de estudio estructural y funcional del cerebro, a finales del siglo XX se comenzó a acceder a la forma en que el cerebro humano se activa y modifica por la experiencia. Como su nombre lo indica, con estos estudios es posible realizar observaciones referidas a la estructura y función de determinadas zonas durante la actividad cognitiva, y observar las modificaciones suscitadas en el cerebro a través de la experiencia.

Estas técnicas permitirían el acceso a formas novedosas de comprender cómo la educación impacta en el cerebro humano, generando numerosos cambios que llevan a la comprensión de los fundamentos neurobiológicos del proceso de enseñanza y aprendizaje.

En este artículo se pretende, en consecuencia, revisar los aportes, límites y caminos futuros de la neuroeducación, entendida como disciplina en formación y no exenta de críticas. Específicamente, se abordarán los estudios sobre plasticidad, neuronas espejo, el aporte del ejercicio físico y las horas de sueño en relación con el aprendizaje. Al final, se realizará un análisis y una conclusión crítica sobre la información revelada. 


\section{INTERVENCIONES DE LAS NEUROCIENCIAS: LA PLASTICIDAD NEURONAL}

Un hito fundamental en el campo de las neurociencias es la noción de plasticidad neuronal, según el cual nuestras redes neuronales se modifican a lo largo de nuestro desarrollo ontogenético. A partir de investigaciones con animales llevadas a cabo a mediados del siglo $X X$, se encontró que la exposición a ambientes complejos o con falta de estimulación sensorial y social, se asocia a diversos cambios estructurales, tales como el número y la forma de los contactos entre neuronas, la expresión genética y la cobertura de mielina en los axones (Lipina, 2016). Se ha descubierto en ratas que los ambientes enriquecidos aumentan en un $25 \%$ las conexiones sinápticas por neurona en las áreas visuales en oposición a los casos de ratas criadas de manera aislada (Greenough, Black \& Wallace, 1987). En el nivel comportamental, por otra parte, se ha hallado en estudios con humanos que los ambientes con mayor estimulación (presencia de libros en el hogar, nivel de educación materna y paterna, tipo de apego, nivel socioeconómico, entre otros) predicen el desempeño de infantes y niños en tareas con demandas cognitivas (Labín, Taborda \& Breñilla, 2015; Richaud \& Arán Filipetti, 2015; Vales, Mora, Martínez, Gómez, Lungo \& Figoli, 2017). El ser humano es producto, por tanto, de sus ambientes y de su disposición innata en simultáneo, surge como una interrelación entre ambos factores. Precisamente, la epigenética se refiere al estudio de cómo los genes producen su efecto en el fenotipo del organismo (Bedregal, Shand, Santos \& Ventura, 2010), y hoy en día se reconoce el papel fundamental que el ambiente extranuclear, extracelular y social ejerce en la modulación de la actividad genética (Shonkoff \& Phillips, 2000). El sujeto realiza una reconstrucción propia a partir de la interacción del genoma con el ambiente, sin darse un determinismo de ninguno de los dos polos, sino que, más bien, la propia subjetividad del individuo está implicada en todo proceso de aprendizaje (Baquero, 1996).

En este marco, es importante tomar en cuenta la noción de período crítico, que alude a un período determinado en el tiempo que permite el desarrollo de una determinada habilidad, como, por ejemplo, la visión y la audición. Luego de este, la adquisición de estas determinadas habilidades, cruciales para el desarrollo posterior, se dificulta profundamente (Cicchetti \& Curtis, 2006). Sin embargo, hay un período sensible de desarrollo, en el que determinadas habilidades tienen mejores oportunidades de ser aprendidas e interiorizadas. En este punto, es interesante mencionar el ya clásico ejemplo de Nico y Brooke descrito por Fischer (2009) e Immordio-Yang (2007). A Brooke 
le fue removido el hemisferio izquierdo a los 11 años, edad problemática para este tipo de cirugía, ya que la habilidad del cerebro para recobrarse de esta intervención severa y adaptarse a los nuevos aprendizajes decrece con el paso del tiempo (Bailey, Bruer, Symons \& Lichtman, 2001). A pesar de que el pronóstico indicaba dificultades permanentes en el habla, con el tiempo Brooke logró volver a utilizar lenguaje oral. Y el avance obtenido en el plano lingüístico se debía, según los autores, al constante apoyo que recibía de su familia y docentes, el cual incluía objetivos específicos como generar habilidades en el lenguaje y el dibujo.

Luego, Immordino-Yang $(2004,2007)$ estudió la capacidad de entonación en Brooke y Nico. A este último, al contrario de Brooke, le había sido removido el hemisferio derecho. De acuerdo con las neurociencias, la capacidad de entonación se encuentra en este hemisferio, con lo cual se predecía que el desempeño de Nico iba a ser más bajo que el de Brooke. Sin embargo, Nico logró desarrollar eficazmente dicha capacidad, lo cual proporcionó una prueba más de la plasticidad cerebral, dado que en su desarrollo se pueden obtener las mismas habilidades a través de procesos disímiles. Justamente, el sistema educativo en general posee una forma masificada de impartir su enseñanza (Cantero, 2013) que no se ajusta a las diversas maneras de aprendizaje de habilidades cognitivas. Por ejemplo, en las personas diagnosticadas con dislexia y discalculia, las formas de aprender lectura y cálculo, respectivamente, difieren de la media, y requieren metodologías de enseñanza particulares (China \& Ferreres, 2016; Fink, 2006; Reigosa-Crespo, González, León, Torres, Mosquera \& Valdés, 2013).

Teniendo en cuenta esto, uno de los aportes más importantes de la neurociencia a la educación lo constituyen las estrategias educativas y terapéuticas que generó en torno a los trastornos del aprendizaje y del desarrollo. Así, hay áreas específicas implicadas en cada uno de los trastornos del aprendizaje, y se han llevado a cabo estrategias de intervención concretas para cada una de ellas. Por ejemplo, en la dislexia se pueden encontrar déficits de percepción, disartrías cerebelosas, lesiones neurológicas, cuadros psicopatológicos asociados, entre otras, que se expresan en omisiones, problemas de comprensión global, lectura silábica, etc. (Fonticiella, 2007).

Teniendo en cuenta esto último, se puede realizar una intervención más ajustada al caso, que dependiendo del momento en que los profesionales intervengan, podrá generar un mejor pronóstico. Lo mismo se ha indagado en trastornos como la discalculia, disgrafía, dislexia, déficit atencional y el 
trastorno del espectro autista (China \& Ferreres, 2016). De esta manera, estos estudiantes pueden integrarse de mejor manera a los entornos educativos. Como afirma Gabrieli (2016), las neurociencias realizan importantes aportes para promover determinadas políticas públicas para paliar las dificultades que la vulnerabilidad social trae aparejada en los primeros estadios del desarrollo.

\section{LAS NEURONAS ESPEJO Y EL APRENDIZAJE}

En el plano de las neurociencias y la educación, también es importante tener en cuenta el concepto de neuronas espejo, que brindan un entendimiento neurobiológico de la empatía y la teoría de la mente. Este tipo de neuronas nos permiten comprender a los demás y nos vinculan con el punto de vista emocional que la otra persona brinda. Las neuronas se activan al realizar cierta acción y al observar las acciones de otras personas. Por ende, se supondría que son las precursoras del aprendizaje por imitación, la interacción del yo y la comprensión social (Pineda-Alhucema, 2016).

Las dos hipótesis que se sostienen con respecto al origen de dichas neuronas son, por un lado, la hipótesis evolutiva (Rizzolatti \& Craighero, 2004), según la cual ellas fueron seleccionadas por la naturaleza, pues eran funcionales para comprender qué hacían los otros. Desde este punto de vista, la capacidad para relacionar acciones observadas y realizadas es innata. Por otro lado, la hipótesis del aprendizaje asociativo (Hickok, 2014; Heyes, 2010) afirma que estas neuronas son resultado de este tipo de aprendizaje y se originarían al asociar la experiencia visual de la acción con su ejecución. Es decir que la capacidad de las neuronas espejo se generaría durante el desarrollo.

Más allá del debate sobre su aparición, hay consenso en que este tipo de neuronas fomentan el aprendizaje de tipo procedural, observacional, imitativo y asociativo (Barrios-Tao, 2016; Catmur \& Heyes, 2017; Hickok, 2014). En una investigación llevada a cabo por Ertelt, Small, Solodkin, Dettmers, McNamara, Binkofski \& Buccino (2007), en pacientes con déficit motor se utilizó entrenamiento físico junto a videos que mostraban diferentes movimientos de acciones cotidianas, hallándose mejorías del grupo que recibió la videoterapia en relación a la línea de base del tratamiento y a un grupo control. Otras investigaciones en rehabilitaciones motrices se llevaron a cabo de manera similar, obteniendo los mismos resultados (Jun-Ding, Yu-Hsuan \& Yu-Wei, 2016). A esto se lo llamó terapia en observación de la acción, y los mismos resultados fueron encontrados para la rehabilitación del lenguaje en pacientes con afasia (Gili, Fiori, De Pasquale, Sabatini, Caltagirone \& Marangolo, 2016). 
Según Meltzoff y Moore (1977), la imitación es una capacidad innata. Estos autores demostraron que los bebés de apenas unos días son capaces de imitar movimientos faciales como abrir la boca o sacar la lengua (conductas que están en el repertorio del recién nacido), lo cual indica que la capacidad para usar equivalencias intermodales está presente desde el nacimiento. Es decir, que el aprendizaje por imitación, promovido por las neuronas espejo, tendría un fuerte impacto desde el nacimiento, de modo que los infantes asignan en su propio cuerpo los comportamientos que observan en los otros con la finalidad de imitarlos. Los mapas somatotópicos serían una parte importante en nuestro desarrollo, ya que facilitan las conexiones que construimos con otras personas, incluso en los primeros meses de vida (Marshall \& Meltzoff, 2015). La habilidad de imitar es así, la base para futuras adquisiciones en el campo del desarrollo social.

Ahora bien, aunque algunos investigadores argumentan que este tipo de neuronas demuestran que somos seres sociales y que estamos diseñados para interactuar unos con otros, siendo ellas una herramienta importante para que los docentes comprendan la génesis de los procesos de imitación y empatía (Morris Ayca, 2014; Rizzolatti \& Craighero, 2004), otros se inclinan por una postura más escéptica y afirman que el descubrimiento de este tipo de neuronas se ha sobregeneralizado a capacidades cognitivas complejas, y realmente no arroja aún luz sobre las formas de comprender las intenciones de los otros que caracterizan a los seres humanos; por tanto, aún se necesitan investigaciones en el contexto áulico, más allá de las realizadas en laboratorios con métodos psicofisiológicos (Gabrieli, 2016; Hickok, 2014; Heyes, 2010).

\section{LAS EMOCIONES EN EL CAMPO EDUCATIVO}

Otro campo fuerte en la neuroeducación es el de las emociones. Egan (2005) ya había tratado este tema en el campo de la pedagogía, y reivindicó la necesidad de revalorar el uso de las emociones en este contexto, comenzando por la imaginación del alumno como disparador para generar nuevas ideas. Sin embargo, este mismo autor muestra que las emociones son relegadas en lo social y esto se acentúa al comenzar el niño la escuela primaria. También, Damasio (1996) afirmó que toda toma de decisión que realizamos, o idea que manejamos en nuestra mente, indefectiblemente está teñida de emoción; por ende, resulta importante su trabajo en el campo educativo.

El estudio internacional de la Fundación Botín (2008), realizado con 500.000 estudiantes de educación infantil, primaria y secundaria, demostró que el 
desarrollo sistemático de programas de educación emocional y social en la escuela repercute positivamente en el bienestar emocional de niños y jóvenes, en sus relaciones sociales, también mejora su rendimiento académico y actúa como factor preventivo de problemas mentales y conductuales en su desarrollo.

Las neurociencias han demostrado que las emociones mantienen la curiosidad, y que las emociones positivas facilitan la memoria y el aprendizaje (Ruetti, Ortega \& González, 2014). Las emociones activan el hipocampo, que está relacionado con la memoria y el aprendizaje, anclando mejor los conocimientos obtenidos, ya que produce recuerdos de tipo emocional con la mediación de la amígdala (LeDoux, 1998), logrando que luego sean más sencillas de evocar (Erk, Kiefer, Grothe, Wunderlich, Spitzer \& Walter, 2003). De acuerdo con Ballarini (2015), hay un entrecruzamiento entre la emoción y la memoria que genera recuerdos fuertemente sostenidos en la memoria a largo plazo, y con mayores posibilidades de ser recuperado. Cuando el cerebro registra las novedades, a través de las fibras nerviosas aumenta durante algún tiempo su capacidad perceptiva general (Fenker, 2009).

Con respecto a este último punto, cabe destacar el impacto de la novedad y la sorpresa positiva en la educación, que genera la liberación del neurotransmisor de la dopamina, generalmente asociada con el sistema de recompensa y placer del cerebro, y con la memoria y atención. En un estudio realizado en animales por Waelti, Dickinson \& Schultz (2001), se analizaron las respuestas de neuronas dopaminérgicas, y se encontró que su activación se da cuando las expectativas con las que se contaban son superadas, demostrando que más que el reforzamiento positivo, importa su calidad y lo inesperado.

\section{EJERCICIO FÍSICO Y SUEÑO}

Otro punto de interés para la neuroeducación es la actividad física, que según los investigadores puede traer aparejada una mejora general de las funciones cognitivas, mayor autoestima, y beneficiar a personas diagnosticadas con TDAH, ansiedad o depresión, además de prevenir el síndrome de Burnout en escolares (Carriedo, 2014).

La reducción del estrés es primordial, ya que cuando es crónico, puede Ilegar a reducir la materia gris de la zona frontal y temporal cerebral, áreas asociadas a las funciones ejecutivas. Además, en este tipo de casos, los niveles de la hormona de cortisol son mayores, afectando la estructura cerebral a nivel epigenético. Esto hace que las dificultades para la regulación 
de la atención y las emociones se profundicen, habilidades nucleares para un buen desempeño escolar (Blair \& Raver, 2016).

Por otro lado, Hanneford (1995), así como Portolés \& González (2016) han determinado los beneficios que puede tener la educación física sobre el rendimiento académico escolar. Y como señalan Blakemore y Frith (2011), el ejercicio también mejora la predisposición física y psicológica hacia el aprendizaje, aumentando los niveles de motivación y atención. A nivel neuronal estos autores encontraron que el ejercicio físico es un neuroprotector de ciertos tipos de células cerebrales y que el estrés celular leve que produce el ejercicio previene el estrés más intenso.

En esta línea, Aberg, Pedersen, Torén, Svartengren, Bäckstrand, Jonson \& Kuhn (2009) realizaron un estudio longitudinal en el cual demostraron que las capacidades cardiovasculares correlacionan con la capacidad intelectual y cognitiva. En concreto, ellos Incluyeron la actividad física al inicio, o la intercalaron con las clases y no al final. También se permitieron recreos al aire libre, observando que en ambos casos se generan mayores grados de motivación y activación (arousal) a lo largo de las clases, mejorando el nivel académico y disminuyendo los niveles de estrés.

En un estudio con animales, Gilestro y colaboradores (2009) hallaron al respecto que dormir adecuadamente también reduce los niveles de estrés y promueve la capacidad memorística. Golombek (2011) echó mano de la disciplina de la cronobiología, ciencia de las neurociencias que estudia los procesos biológicos que siguen unas secuencias temporales previsibles, aplicándolos al estudio de los procesos educativos. Para esto acuñó el concepto de cronoeducación, que se propone investigar los ritmos propios de sueño y vigilia con la intención de mejorar la calidad de enseñanza y aprendizaje. En este sentido, el tiempo y la temporalidad biológica son variables que relacionan la neurociencia con el mundo de la enseñanza y el aprendizaje (Louzada \& Mena-Barreto, 2007).

El reloj interno de cada individuo, también llamado ritmo circadiano, está asociado a los cambios de luz-oscuridad que se dan durante el día y la noche. Cuando los hábitos del sueño se ven desregulados pueden surgir trastornos del sueño, como suele ocurrir con los adolescentes. La glándula pineal, por su parte, segrega una hormona (melatonina) que actúa como un código químico nocturno y es una poderosa ayuda para regular estos desfases (Battro, 2011). 
En general, los adolescentes suelen acostarse tarde y el cuerpo se adapta rápidamente a este cambio, pero no cuando debe volver a despertar temprano. El adolescente funciona mejor en horarios nocturnos, y eso se da no solamente por cuestiones sociales, sino también por el horario que les marca su reloj biológico (Golombek, 2011; Roenneberg \& Merrow, 2014).

Este cronotipo vespertino entra en conflicto con los horarios matutinos habituales y afecta su calidad de vida, su salud, desempeño académico y su capacidad cognitiva, generando una disociación que algunos autores Ilaman Jet lag social (Wittmann et al., 2006). Golombek (2011), Roenneberg \& Merrow (2014) y Wittmann et al., (2006) argumentan por ello que es necesario que las materias que demandan menos recursos cognitivos se ubiquen al principio del horario, mientras que las más complejas se ubiquen por la mitad. Además, se debe realizar psicoeducación con respecto a los temas de la cronobiología y los trastornos del sueño, para que los estudiantes comprendan la importancia de dormir correctamente.

\section{LIMITACIONES Y CAMINOS FUTUROS EN NEUROEDUCACIÓN}

Como ya fue afirmado, entre las neurociencias podemos vislumbrar a la neuroeducación, disciplina que se encarga de aplicar sus más recientes descubrimientos neurocientíficos al mejoramiento de los procesos educativos de enseñanza y de aprendizaje (Battro, 2011). Como su nombre lo indica, genera puentes de conocimiento y encuentro entre las neurociencias y la educación; sin embargo, esta idea no está exenta de críticas. Bruer (1997) indica, por ejemplo, que el puente entre ambas disciplinas aún es "muy lejano", ya que muchos de los aportes de las neurociencias se realizan con base en experimentos en animales y difícilmente son extrapolables a los humanos. Por ende, hay que ser cautelosos a la hora de realizar afirmaciones en lo relativo a los aportes de la neuroeducación.

En este sentido, hay investigaciones que concluyen que las explicaciones de fenómenos psicológicos tienden a generar mayor interés público cuando contienen información neurocientífica, independientemente de la lógica de las explicaciones (Fernandez-Duque, Evans, Chrisrtian \& Hodges, 2015; Skolnick, Keil, Goodstein, Rawson \& Gray, 2009). En los trabajos antes citados se entregó una breve descripción sobre fenómenos psicológicos, seguida de cuatro tipos de explicaciones, con un diseño de dos (buenas explicaciones/ malas explicaciones) por dos (con neurociencias/sin neurociencias). Salvo los sujetos del grupo de expertos, los demás juzgaron que las explicaciones que poseían el contenido neurocientífico eran más satisfactorias. Los autores 
concluían que se deja de lado la mirada crítica con respecto al tema, lo que impacta de forma notable en el juicio de los que no poseen experiencia en el tema.

También se debe tener en cuenta cómo divergen las situaciones de laboratorio con los contextos escolares, ya que conservar la validez ecológica en este tipo de investigaciones es realmente complicado. Justamente, esto es lo que Daniel y Poole (2009) llamaron ecología pedagógica. Para enriquecer los resultados y para generar estrategias factibles de ser aplicadas, las investigaciones deben ser realizadas en ambos contextos (Fischer, 2009; Howard Jones, 2011).

Otras críticas, realizadas por Bowers (2016) y De Vos (2016), enfatizan que las neurociencias no aportan ningún conocimiento práctico al área educacional más allá del conocimiento de la psicología cognitiva y del comportamiento. Según estos autores, las neurociencias no han aportado propuestas educativas específicas y en nada pueden ayudar a los educadores, pero sí se podría dar el caso contrario. Además, argumentan que las teorías que por el momento fueron aportadas desde el campo son auto-evidentes, y cita las relacionadas con las dificultades de aprendizaje relacionados con entornos estresantes, malos hábitos de sueño y falta de ejercicio. Mencionan, asimismo, que las explicaciones neurocientíficas sobre los cambios en el cerebro encontradas en los niños en situaciones educativas tienen poco valor práctico con respecto a lo que aprenden y expresan en sus comportamientos. Por último, resaltan la importancia del estudio comportamental para seguir avanzando en el área educativa y en la comprensión de los procesos de enseñanza y aprendizaje.

También Dekker, Lee, Howard Jones \& Jolles (2012) consideran que hay muchas concepciones erradas por parte de los profesionales de la educación en relación con el cerebro. A las mismas se las llama "neuromitos", y en una muestra de 242 profesores de escuelas primarias y secundarias interesados en las neurociencias aplicadas a la educación, se encontró que el $49 \%$ de dichos neuromitos fueron corroborados. De estos fueron: "Solo usamos el $10 \%$ del cerebro", "Los problemas de aprendizaje asociados con diferencias en el desarrollo de funciones cerebrales no pueden ser remediados por la educación", "Hay períodos críticos durante la niñez, luego de los cuales algunos aprendizajes ya no pueden lograrse", entre otros (Dekker et al., 2012). Según los investigadores, estos hallazgos sugieren que los docentes entusiastas de la aplicación de las neurociencias a la educación no distinguen con facilidad entre pseudociencia y hechos científicos. Incluso, los más 
instruidos en el tema de las neurociencias, eran los que más confirmaban los neuromitos. Esto demuestra la necesidad de ampliar el trabajo interdisciplinario y de generar intervenciones basadas en evidencia empírica. Como se mencionaba anteriormente, la neuroeducación es una disciplina aún en construcción, y no se debe dejar de ser críticos con sus aportes.

En relación con esto, Satel y Lilienfeld (2013) revelan cómo muchas de las aplicaciones de la neurociencia humana al mundo real, muchas veces pasan por alto sus limitaciones y complejidades, y llegan a posturas reduccionistas que no dejan ver la complejidad de factores que dan forma a nuestro comportamiento e identidades. Según estos autores, los escáneres cerebrales son representaciones útiles, pero a menudo ambiguas de un sistema muy complejo. Cada región del cerebro participa en una multitud de experiencias simultáneas e interactúa con otras regiones, por lo que ver una luz en la superficie de un resonador magnético funcional en respuesta a un estímulo, no indica automáticamente una sensación particular y menos explican la comprensión de las funciones cognitivas superiores que provienen de esas interacciones. Como Satel y Lilienfeld proponen, este punto de vista "neurocéntrico" de la mente puede socavar una mirada amplia del comportamiento y la experiencia, que debiera ser analizada en distintos niveles.

Sin embargo, para Marcus (2013), aunque todavía estamos lejos de que se conviertan en la verdad última, tampoco debemos ser pesimistas con los resultados de las neurociencias. Este autor enfatiza la importancia de trabajar con campos como la psicología y la psiquiatría, que tienen como punto de partida la mente (en lugar del cerebro) para complementar la neurociencia.

Los elementos básicos de la psicología, como creencias, deseos, metas y pensamientos, juegan un papel clave en la comprensión de la conducta humana, por lo que la ciencia necesita de investigadores que estudien la mente, al igual que investigadores que estudien el cerebro. Nuestro objetivo no debe limitarse a recoger el cerebro desde la mente o viceversa, sino trabajar de forma simultánea con todas las disciplinas para construir puentes más sólidos entre nuestro entendimiento de ambos (Marcus, 2013).

En la misma postura se encuentra Gabrieli (2016), quien afirma que la neurociencia educacional debe ser entendida como una ciencia básica que puede ser utilizada luego en la práctica escolar cotidiana e impactar en las políticas públicas para ayudar a estudiantes de sectores vulnerables. En tanto que Howard-Jones, Varma, Ansari, Butterworth, De Smedt, Goswami \& Thomas 
(2016) consideran que la neuroeducación es una interdisciplina que genera puentes entre las neurociencias cognitivas, la psicología cognitiva y la práctica educativa. En consecuencia, las prácticas de la psicología deben ir de la mano con las de la neurociencia, y no se pueden pensar como disciplinas separadas.

La neuroeducación, según Stringer \& Tommerdahl (2016), seguirá creciendo de la mano del crecimiento tecnológico. Estos autores plantean que, con el avance de las investigaciones en el campo de la educación y las neurociencias, recolectando datos sobre la edad, el tipo de didáctica y pedagogía utilizada por parte de los docentes y directivos, el tipo de contenidos enseñado, entre otros, se generarán modelos capaces de predecir los resultados de aprendizaje, representando sistemas complejos en interacción. Esto se realizaría a partir de simulaciones virtuales de grupos enteros de clases, en los que experimentar con estrategias políticas y alternativas de enseñanza, puede convertirse en una poderosa herramienta para anticipar lo que funciona antes de probar las intervenciones sobre los niños reales.

Todavía faltan algunos años para que estos modelos predictivos sean válidos y confiables, pues a pesar de que hay datos fáciles de obtener y ya se encuentran disponibles (como la edad de los estudiantes, su etnia, lengua nativa, clases tomadas, puntajes en tests y las tazas de finalización), otros, ligados a la historia personal todavía se están buscando en estudios longitudinales, y permitirían analizar cómo interactúan los ambientes particulares y los resultados del aprendizaje en un niño individual. Así, los modelos predictivos podrán asistir a padres, terapeutas y profesores en la selección de intervenciones específicas para cada individuo particular (Stringer \& Tommerdahl, 2016).

En Japón, el equipo de Koizumi (2005) va más allá de la práctica en el laboratorio y analiza el funcionamiento cerebral a través de máquinas livianas y portátiles de topografía óptica en contextos áulicos. Este equipo lleva a cabo un estudio neurocognitivo de carácter longitudinal con 10.000 niños, en el cual se espera obtener unas 1000 imágenes funcionales del cerebro infantil en diferentes momentos de su maduración y escolaridad (Konishi, 2005). Todos estos avances y cambios en el contexto de la neuroeduación acarrean dilemas éticos que son trabajados por la neuroética (Sheridan, Zinchenko \& Gardner, 2005).

A pesar del avance en la tecnología, autores como Samuels (2009) y Ravet y Williams (2016) piensan que hay una oposición histórica de carácter metodo- 
lógica, epistemológica y ontológica entre las neurociencias y la educación. Mientras la primera posee una tradición cuantitativa, la segunda se mantiene en una tradición de corte cualitativo e interpretativo. Según estos autores, para generar un puente más fuerte entre ambas disciplinas en un futuro, deberán utilizarse metodologías mixtas, utilizando un marco teórico basado en una epistemología pluralista.

\section{CONCLUSIONES Y DISCUSIÓN}

El presente artículo tenía por objetivo revisar aportes de las neurociencias al campo educativo, así como las limitaciones y direcciones futuras de la reciente disciplina de la neuroeducación. A partir de la información revelada se puede concluir, por un lado, que las neurociencias han aportado al campo educativo el estudio de las dificultades del aprendizaje y han generado métodos aplicables para paliarlas a la hora de aprender (China \& Ferreres, 2016; Fischer, 2009; Immordino-Yang, 2007; Lipina, 2016). Las formas de aprender varían según cada dificultad específica en el aprendizaje y ello genera políticas públicas basadas en la inclusión de estudiantes con dificultades de aprendizaje (Gabrieli, 2016).

Por otra parte, las neurociencias han arrojado luz al conocimiento relativo a los momentos de intervención, mediante el concepto de período sensible, que no permite caer en el neuromito del período crítico, según el cual, pasado cierto momento en el desarrollo, si una habilidad no ha sido desarrollada, luego será imposible rehabilitarla. En su lugar, habría períodos sensibles que deben ser estimulados para que determinada capacidad pueda desarrollarse de forma óptima, pero también puede ser aplicada la rehabilitación cognitiva, que gracias a la plasticidad neuronal, puede generar nuevas capacidades y rehabilitar las que fueron perdidas (Cicchetti \& Curtis, 2006). Más aún, esto cobra una importancia cabal en estadios tempranos del desarrollo, cuando se generan las bases para futuras adquisiciones (China \& Ferreres, 2016; Gabrieli, 2016).

Otro aporte de las neurociencias se relaciona con los entornos enriquecidos, que generan un desarrollo más propicio de las capacidades cognitivas (Greenough, Black \& Wallace, 1987; Richaud \& Arán Filippetti, 2015). En tal sentido, es necesario generar un ambiente enriquecido para que su aprendizaje sea significativo. Con respecto a esto, desde las neurociencias se apunta a generar entornos educativos que utilicen las emociones y la novedad, ya que las mismas estarían asociadas a mayores niveles atencionales, de aprendizaje y de memoria (Ballarini, 2015; Ruetti, Ortega \& González, 2014). 
También, las neurociencias han aportado el concepto de neuronas espejo, que permite comprender la génesis de los procesos de imitación e interacción, y el fundamento neurológico del aprendizaje observacional, procedural, imitativo y asociativo (Barrios-Tao, 2016; Catmur \& Heyes, 2017; Hickok, 2014).

Por último, el tema de la importancia de la actividad física y el sueño adecuado desarrollado por las neurociencias debería ser más implementado en las instituciones educativas, ya que promueve la salud mental, física, cerebral y cognitiva (Blakemore y Frith, 2011; Golombek, 2011).

Se debe tener en cuenta que las neurociencias se encuentran en pleno crecimiento y expansión, generando modelos predictivos e investigaciones con crecientes niveles de validez ecológica. Sin embargo, no se debe caer en una postura reduccionista e intentar comprender, mediante ellas, toda la complejidad que implica el comportamiento humano a través del cerebro. Todavía son muchos los avances que deben lograr las neurociencias de la educación para perfeccionar y ampliar su aplicación al campo.

En esta última línea, se encontraron autores escépticos (Bowers, 2016; De Vos, 2016; Bruer, 1997), para quienes las neurociencias aún no realizan aportes para el campo educativo, y que sus entrecruzamientos están lejos de llevarse a cabo.

Finalmente, muchos otros autores mantienen una actitud optimista con respecto a los aportes actuales y futuros de la disciplina al campo educativo (Gabrieli, 2016; Howard-Jones et al., 2016; Marcus, 2013; Stringer y Tommerdahl, 2016). Desde este punto, las neurociencias deben seguir trabajando con el conjunto de las disciplinas que la conforman para generar una mirada abarcadora de los procesos de enseñanza y aprendizaje del ser humano. Para esto, debe comprender el comportamiento a través de distintos niveles de análisis como son el molecular, neuronal, psicológico y social. Se deben realizar debates, investigaciones novedosas y artículos científicos directamente aplicables a la realidad educativa, que dejen marcas en los enseñantes para que puedan ser aplicadas (Satel y Lilinfield, 2013).

Sin embargo, se debe ser crítico con la información aportada por la neuroeducación, ya que no solo se suele caer en concepciones erróneas sobre sus aportes (Dekker et al., 2012; Skolnick et al., 2009), sino que muchas veces no tienen en cuenta la validez ecológica (Daniel \& Poole, 2009; Fischer, 2009; Howard-Jones, 2011). Para esto deben realizarse entornos interdisciplinarios 
de trabajo, que conjuguen diferentes conocimientos puestos a disposición de los procesos de enseñanza y aprendizaje. Es necesario generar intervenciones basadas en la evidencia y seguir produciendo investigaciones que puedan aplicarse al campo de la educación. En este aspecto se propone enfatizar el trabajo interdisciplinario de la psicología y específicamente las ciencias cognitivas y del comportamiento, que ya han mostrado resultados favorables en este campo.

De los aportes recabados y de las limitaciones revisadas se desprende también que muchas investigaciones fueron hechas con animales, y aún queda extrapolar sus resultados en seres humanos. Además, solo muy pocas fueron realizadas en entornos educativos. Teniendo en cuenta, entonces, que la neuroeducación es una disciplina en expansión, se debe ser cauto y crítico con los resultados hallados en este campo, y fomentar investigaciones con mayor validez ecológica.

En síntesis, este artículo es una invitación para que investigadores de diferentes disciplinas y docentes de diferentes asignaturas debatan y se enriquezcan a partir de los entrecruzamientos de los distintos conocimientos, pues, como se ha reiterado, los puentes que se pueden trazar entre neurociencias y educación están aún en construcción, y son necesarios más trabajos novedosos con metodologías plurales para lograr una fundamentación contundente sobre su aplicación.

Agradecimientos: Este trabajo fue parcialmente financiado por la Agencia Nacional de Promoción Científica y Tecnológica y por la Universidad Abierta Interamericana.

Nota de autores: Este artículo fue realizado en el marco del proyecto subsidiado UAI "Desarrollo temprano integrado de competencias cognitivas y de comunicación: Influencia de factores individuales y ambientales", y del Proyecto AGENCIA PICT 2013-2467 "Desarrollo de la comunicación y procesos ejecutivos en niños. Temperamento y factores socioeconómicos".

\section{REFERENCIAS}

Aberg, M., Pedersen, N., Torén, K., Svartengren, M., Bäckstrand, B., Jonson \& Kuhn., G. (2009). Cardiovascular fitness is associated with cognition in young adulthood. PNAS, 106(49), 20906 -20911. https://doi.org/10.1073/pnas.0905307106

Bailey, D. B., Jr., Bruer, J. T., Symons, F. J. \& Lichtman, J. W. (2001). Critical thinking about critical periods. Baltimore: Paul H. Brookes.

Ballarini, F. (2015). REC. Por qué recordamos lo que recordamos y olvidamos lo que olvidamos. Buenos Aires: Editorial Sudamericana. 
Baquero, R. (1996). Ideas centrales de la Teoría Socio Histórica. En R. Baquero. Vigotsky y el aprendizaje escolar (pp. 31-62). Buenos Aires: Aique Grupo Editor S.A.

Barrios-Tao, H. (2016). Neurociencias, educación y entorno sociocultural. Educación y Educadores, 19(3), 395-415. https://doi.org/10.5294/edu.2016.19.3.5

Battro, A. M. (2011). Neuroeducación: El cerebro en la escuela. En La Pizarra de Babel: Puentes entre neurociencia, psicología y educación. S. Lipina y M. Sigman (Editores). Buenos Aires: El Zorzal.

Battro, A. M. \& Cardinali, D. P. (1996). Más cerebro en la educación. Buenos Aires: La nación

Bedregal, P., Shand, B., Santos, M. \& Ventura, P. (2010). Aportes de la epigenética en la comprensión del desarrollo del ser humano. Revista médica de Chile, 138(32), 366- 372. https://doi.org/10.4067/S0034-98872010000300018.

Blair, C. \& Raver, C. (2016). Poverty, Stress, and Brain Development. Academic Pediatrics, 16(3), 23-31. https://doi.org/10.1001/jamapediatrics.2015.1475

Blakemore, S. J. \& Frith, U. (2011). Cómo aprende el cerebro. Las claves para la Educación. Ariel: Barcelona.

Bowers, J. S. (2016). The Practical and Principled Problems With Educational neuroscience. Psychological Review, 4(2), 27-36. https://doi.org/10.1037/rev0000025

Bruer, J. T. (1997). Education and the brain: A Bridge too Far. Educational Researher, 26(8), 4-16. https://doi.org/10.3102/0013189X026008004

Cantero, M . (2013). La educación para la muerte. Un reto formativo para la sociedad actual. Psicogente, 16(30), 424-438.

Carriedo, A. (2014). Beneficios de la Educación Física en alumnos diagnosticados con Trastorno por Déficit de atención con Hiperactividad (TDAH). Journal of Sport and Health Research, 6(1), 47-60.

Catmur, C. \& Heyes, C. (2017). Mirroring "meaningful" actions: sensorimotor learning modulates imitation of goal-directed actions. The Quarterly Journal of Experiment Psychology, 9(14), 1-38. http://dx.doi.org/10.1080/17470218.2017.1344257

China, N. \& Ferreres, A. (2016). Cerebro, lectura y dislexia. En A. Ferreres \& V. Abusama, (Eds.), Neurociencias y educación. Buenos Aires: Paidós.

Cicchetti, D. \& Curtis, J. (2006). The developing brain and neural plasticity: implications for normality, psychopathology and resilience. Developmental Psychopathology, 2(7), 52-64. https://doi.org/10.1016/j.chiabu.2011.10.006

Damasio, A. (1996). El error de Descartes. La razón de las emociones. Santiago de Chile: Andrés Bello.

Daniel, C. \& Poole, A. (2009). Learning for life, an ecological approach to pedagogical research. Perspectives on Psychological Science, 4(1), 91- 96. https://doi. org/10.1111/j.1745- 6924.2009.01095.x.

De Vos, J. (2016). The Educated Brain: A Critique of Neuroeducation. De Vos, J. (Eds.), The Metamorphoses of the Brain-Neurologisation and its Discontents. Ghent: palgrave MacMillan.

Dekker, S., Lee, N., Howard Jones, P. \& Jolles, J. (2012). Neuromyths in Education: Prevalence and Predictors of Misconceptions among Teachers. Frontiers in Psychology, 3(2), 415- 429. https://doi.org/10.3389/fpsyg.2012.00429

Egan, K. (2005). ¿Empezar desde lo que el alumno sabe o desde lo que el alumno puede imaginar? Revista de didáctica de la lengua y la literatura, 3(2), 15-23. 
Erk, S., Kiefer, M., Grothe, J., Wunderlich, A., Spitzer, M. \& Walter, H. (2003). Emotional context modulates subsequent memory effect. Neuroimage, 2(4), 439- 447. https://doi.org/10.1016/S1053-8119(02)00015-0

Ertelt, D., Small, S., Solodkin, A., Dettmers, C., McNamara, A., Binkofski, F. \& Buccino, G. (2007). Action observation has a positive impact on rehabilitation of motor deficits after stroke. Neurolmage, 36(8), 164-173. https://doi.org/10.1016/j. neuroimage.2007.03.043

Fenker, D. (2009). Importancia de la novedad en el aprendizaje y la memoria. Mente y Cerebro, 36(41), 21-36.

Fernandez-Duque, D., Evans, J., Chrisrtian, C. \& Hodges, S. (2015). Superfluous Neuroscience Information Makes Explanations of Psychological Phenomena More Appealing. Journal of Cognitive Neuroscience 27(5), 926-944. https://doi. org/10.1162/jocn_a_00750

Fink, R. P. (2006). Why Jean and John couldn't read and how they learned?. Newark, DE: International Reading Association.

Fischer, K. (2009). Mind, Brain and Education; Building a Scientific Groundwork for Learning and Teaching. Mind Brain and Education, 3(1), 3-16. https://doi. org/10.1111/j.1751-228X.2008.01048.x

Fonticiella, S. (2007). Trastornos del aprendizaje: Enfoque desde las Neurociencias. Educación inclusiva, 4(2), 13-30.

Fundación Botín. (2008). Educación, emoción y Social. Análisis Internacional. Cantabria, España: Fundación Botín.

Gabrieli, J. (2016). The Promise of Educational Neuroscience: Comment on Bowers. Psychological Review, 123(5), 613-619. http://dx.doi.org/10.1037/rev0000034

Gilestro, G., Tononi, G. \& Cirelli, C. (2009). Widespread Changes in Synaptic Markers as a Function of Sleep and Wakefulness in Drosophila. Science, 324(3), 109-112. https://doi.org/10.1126/science.1166673

Gili, T., Fiori, V., De Pasquale, G., Sabatini, U., Caltagirone, C. \& Marangolo, P. (2016). Right sensory-motor functional networks subserve action observation therapy in aphasia. Brain Imaging and Behavior, 3(2), 1-15. https://doi.org/10.1016/j. neurobiolaging.2016.03.005

Golombek, D. (2011). Cronoeducación: Un tiempo para sembrar, un tiempo para cosechar, un tiempo para aprender. En La Pizarra de Babel: Puentes entre neurociencia, psicología y educación. S. Lipina y M. Sigman (Editores). Buenos Aires: El Zorzal.

Greenough, T., Black, E. \& Wallace, C. (1987). Experience and brain development. Developmental Psychobiology, 22(3), 727-252. https://doi.org/10.2307/1130197

Hanneford, C. (1995). Smart Moves: Why Learning Is Not All in Your Head? Great Ocean Publishers, 7(8), 34-46.

Heyes, C. (2010). Where do mirror neurons come from? Neuroscience and Biobehavioral Reviews, 34(12), 575-583. https://doi.org/10.1016/j.neubiorev.2009.11.007

Hickok, G. (2014). The Myth of Mirror Neurons. The real Neuroscience of Communication and Cognition. Nueva York: Norton \& Company.

Howard, J. (2011). Problemas en la integración neurociencia-educación: acercamiento a la investigación neuroeducacional. En La Pizarra de Babel: Puentes entre neurociencia, psicología y educación. S. Lipina y M. Sigman (Editores). Buenos Aires: El Zorzal.

Howard-Jones, P., Varma, S., Ansari, D., Butterworth, B., De Smedt, B., Goswami, U., Thomas, M., (2016). The Principles and Practices of Educational Neuroscience: 
Comment on Bowers. Psychological Review, 123(5), 620-627. http://dx.doi. org/10.1037/rev0000036

lacoboni, M. (2009). Las neuronas espejo: empatía, neuropolítica, autismo, imitación o de cómo entendemos a los otros. Buenos Aires: Editorial Katz.

Immordino-Yang, M. H. (2004). A tale of two cases: Emotion and prosody after hemispherectomy. Brain and Neurosciences Special Interest Group, 9(3), 32-41.

Immordino-Yang, M. H. (2007). A tale of two cases: Lessons for education from the study of two boys living with half their brains. Mind, Brain, and Education, 1(2), 66-83. https://doi.org/10.1111/j.1751-228X.2007.00008.x

Jun-Ding, Z., Yu-Hsuan, L. \& Yu-Wei, H. (2016). Treatment Effects of Action Observation Therapy in Stroke Rehabilitation: A Systematic Review and Meta-Analysis. Phisical Medicine and Rehabilitation, 97(10), 142- 160. http://dx.doi.org/10.1016/j. apmr.2016.08.441

Koizumi, H. (2005). Brain-Science \& Education. Programs at the Japon Science and Technology Agency (JST). En Brain, Science and Education. Saitama, Japan: Science and Technology Agency.

Konishi, Y. (2005). Introduction of the Cohort Study. En Brain, Science and Education. Saitama, Japan: Science and Technology Agency.

Labín, A., Taborda, A. \& Brenlla, M. (2015). La relación entre el nivel educativo de la madre y el rendimiento cognitivo infanto-juvenil a partir del WISC-IV. Psicogente, 18(34), 293-302. http://doi.org/10.17081/psico.18.34.505

LeDoux, J. E. (1998). Emotion and the limbic system concept. Concepts Neuroscience, 2(1), 169-199.

Lipina, S. (2016). Pobre Cerebro. Buenos Aires: Siglo XXI.

Louzada, F. \& Menna- Barreto, L. (2007). O Sono Na Sala De Aula. Tempo Escolar E Tempo Biológico. Río de Janeiro: Viera \& Lent.

Marcus, G. (2013). The Problem with the Neuroscience Backlash. The New Yorker. Recuperado de: http://www.newyorker.com/tech/elements/the-problem-with-the-neuroscience-backlash

Marshall, P. J. \& Meltzoff, A. N. (2015). Body maps in the infant brain. Trends in Cognitive Sciences, 19(9), 499-505. https://doi.org/10.1016/j.tics.2015.06.012

Meltzoff, A. N. \& Moore, M. K. (1977). Imitation of facial manual gestures by human neonates. Science, 198(43), 75-78. https://doi.org/10.1126/science.198.4312.75

Morris Ayca, M. V. (2014). La neuroeducación en el aula: neuronas espejo y la empatía docente. La vida y la historia, 2(3), 7-18.

Paterno, R. (2014). Luces y penumbras de la neuroeducación. Revista lberoamericana de Psicomotricidad y Técnicas Corporales, 4(3), 22-23.

Pineda-Alhucema, W. F. (2016). La Teoría de la mente desde el marco de la neurociencia cognitiva social. Psicogente, 19(35), 8-10. http://doi.org/10.17081/ psico.19.35.1202

Portolés, A. \& González, J. (2016). Actividad física y niveles de burnout en alumnos de la E.S.O. Retos, 29(4), 95-99.

Ravet, J. \& Williams, J. (2016). What we know now: education, neuroscience and transdisciplinary autism research. Educational Research, 59(1), 1-16. http:// dx.doi.org/10.1080/00131881.2016.1272429

Reigosa-Crespo, V., González, E., León, T., Torres, R., Mosquera, R. \& Valdés (2013). Numerical Capacities as Domain-Specific Predictors beyond Early Mathematics Learning: A Longitudinal Study. PLoS ONE, 8(11), 13-28. https://doi.org/10.1371/ journal.pone.0079711 
Richaud, M. C. \& Arán Filippetti, C. (2015). Desarrollo cognitivo de niños en vulnerabilidad social: una experiencia de intervención. Journal of Psychology Research, 12(5), 684-692. https://doi.org/10.17265/2159-5542/2015.12.003

Rizzolatti, G. \& Craighero, L. (2004). The mirror neuron system. Annual Review of Neuroscience, 27(31), 169-192. https://doi.org/10.1146/annurev. neuro.27.070203.144230

Roenneberg, T. \& Merrow, M. (2014). Entrainment of the Human Circadian Clock. Cold Spring Harbor Symposia on Quantitative Biology, 72(1), 293-299. https://doi. org/10.1101/sqb.2007.72.043

Ruetti, E., Ortega, I. S. \& González, J. M. (2014). Emociones y cognición. Factores moduladores del aprendizaje y la memoria en niños y adolescentes. Rodríguez L. A. y Edson Jorge H. (Eds.), Pensando la psicología educativa en la sociedad del conocimiento. Lima: Universidad Nacional de Educación.

Samuels, B. M. (2009). Can the Differences between Education and Neuroscience be overcome by Mind, Brain and Education? Mind Brain \& Education 3(1), 45-55. https://doi.org/10.1111/j.1751-228X.2008.01052.x

Satel, S. y Lilienfeld, S. (2013). Brainwashed: The Seductive Appeal of Mindless Neuroscience. Washington: Basic Book.

Sheridan, K., Zinchenko, E. \& Gardner, H. (2005). Neuroethics in Education. En J. Illes (Ed) Neuroethics in the 21st Century: Defining the Issues in Theory, Practice and Policy. Oxford: Oxford University Press.

Shonkoff, P. \& Phillips, D. (2000). (Editors) From Neurons to Neighborhoods. The Science of Early Childhood Development. Washington DC: National Academy Press.

Skolnick, D., Keil, F., Goodstein, J., Rawson, E. \& Gray, J. (2009). The seductive Allure of Neuroscience Explanations. Cognitive Neuroscience, 20(3), 470-477. https:// doi.org/10.1162/jocn.2008.20040

Stringer, S. \& Tommerdahl, J. (2016). Building Bridges between Neuroscience, Cognition and Education with Predictive Modeling. Mind, Brain, and Education, 9(2), 121-126. https://doi.org/10.1111/mbe.12076

Vales, L., Mora, B., Martínez, J., Gómez, C., Lungo, R. \& Figoli, I. (2017). ¿La impulsividad cognitiva dificulta el desarrollo de la teoría de la mente en niños en situación de vulnerabilidad social? Revista Colombiana de Psiquiatría, 3(5), 211-234. https://doi.org/10.1016/j.rcp.2017.03.003

Waelti, P., Dickinson, A. \& Schultz, W. (2001). Dopamine responses comply with basic assumptions of formal learning theory. Nature, 412(6842), 37-49. https://doi. org $/ 10.1038 / 35083500$

Wittmann, M., Dinich, M., Merrow, M. \& Roenneberg, T. (2006). Social Jetlag: Misalignment of Biological and Social Time. Chronobiology Internacional, 23(2), 497-509. http://dx.doi.org/10.1080/07420520500545979 autor o el licenciante.

\section{(cc) BY}

